\title{
Effect of acid suppressants on the efficacy of tyrosine kinase inhibitors in patients with epidermal growth factor receptor-mutated non-small-cell lung cancer
}

\author{
KUNIHIKO MIYAZAKI ${ }^{1}$, SHINYA SATO ${ }^{1}$, TAKAHIDE KODAMA ${ }^{1}$, TOMOHIRO TAMURA ${ }^{2}$, \\ KATSUNORI KAGOHASHI ${ }^{3}$, HIROAKI SATOH ${ }^{3}$ and NOBUYUKI HIZAWA ${ }^{2}$
}

${ }^{1}$ Ryugasaki Saiseikai General Hospital, Ryugasaki, Ibaraki $135-0033 ;{ }^{2}$ Faculty of Medicine, Institute of Clinical Medicine,
University of Tsukuba, Tsukuba 305-8575; ${ }^{3}$ Division of Respiratory Medicine, Mito Medical Center,
University of Tsukuba Hospital, University of Tsukuba, Mito, Ibaraki 310-0015, Japan

Received September 3, 2015; Accepted February 22, 2016

DOI: $10.3892 / \mathrm{mco} .2016 .810$

\begin{abstract}
This retrospective study was conducted to evaluate whether oral acid suppressant (AS) therapy is associated with decreased efficacy of gefitinib and erlotinib, particularly in patients with mutated epidermal growth factor receptor (EGFR). A total of 46 consecutive patients with pathologically confirmed non-small-cell lung cancer (NSCLC), who were treated with tyrosine kinase inhibitors (TKIs) in two tertiary hospitals between September, 2005 and May, 2013, were retrospectively analyzed. Of the 46 patients, 11 received AS treatment. As regards age, gender, smoking history, performance status, histology of lung cancer, clinical stage, body surface area (BSA) and type of EGFR mutation, there were no statistically significant differences between patients with and those without AS treatment. There was no statistically significant difference in progression-free survival (PFS) or overall survival (OS) between the two groups of patients ( $\mathrm{P}=0.296$ and 0.613 , respectively). As regards the relative dose of TKI/BSA and survival in patients with and those without AS treatment, there were no statistically significant differences in PFS and OS between the two groups of patients. Our study indicates that AS treatment may not compromise TKI efficacy (gefitinib or erlotinib) in NSCLC patients with mutated EGFR. Prospective studies and large-scale confirmation studies investigating the effect of AS co-administration with TKIs in patients with mutated EGFR may be meaningful in clinical practice.
\end{abstract}

Correspondence to: Professor Hiroaki Satoh, Division of Respiratory Medicine, Mito Medical Center, University of Tsukuba Hospital, University of Tsukuba, 3-2-7 Miya-Machi, Mito, Ibaraki 310-0015, Japan

E-mail: hirosato@md.tsukuba.ac.jp

Key words: acid suppressants, epidermal growth factor receptor tyrosine kinase inhibitors, efficacy, non-small-cell lung cancer

\section{Introduction}

As gastroesophageal reflux disease is highly prevalent among the elderly (1) and peptic ulcer and diffuse erosive gastritis are not uncommon among cancer patients who are under severe mental stress (2), acid suppressant (AS) therapy with a histamine type-2 receptor antagonist (H2RA) and/or a proton pump inhibitor (PPI) are usually prescribed in these patients. Although not common, dyspepsia and abdominal discomfort are two of the possible adverse effects of epidermal growth factor receptor tyrosine kinase inhibitors (EGFR-TKIs) (3). Therefore, drug interactions between AS and TKIs are a clinical issue in the management of patients treated with TKIs. During preclinical evaluation, one of the TKIs, erlotinib, was found to exhibit $\mathrm{pH}$-dependent solubility, with a dissociation constant of 5.4 (3). This pH-dependent solubility was reflected in a study that compared its plasma concentrations in healthy subjects who were or were not receiving AS therapy (4). In addition, the H2RA ranitidine decreased the median area under the curve (AUC) of erlotinib by 33\% (3). Recently, two clinical evaluations of the interaction between AS therapy and erlotinib have been reported, and they reported opposing findings, namely that AS therapy was associated with decreased erlotinib efficacy (5) and that there was no such an association (6). These studies included patients with unknown EGFR mutation status and only evaluated the efficacy of erlotinib therapy $(5,6)$. On the other hand, however, Ter Heine et al reported lower than expected erlotinib trough concentrations in a patient who received pantoprazole, an intravenous PPI, but this discrepancy was not observed when oral pantoprazole was prescribed (7). The EGFR mutation status was not described in that case report.

In the present study, we investigated whether oral AS therapy is truly associated with decreased efficacy of gefitinib and erlotinib, particularly in patients with mutated EGFR.

\section{Patients and methods}

Patients. A total of 46 consecutive patients with pathologically confirmed non-small-cell lung cancer (NSCLC), who were treated with TKIs at the Ryugasaki Saiseikai General Hospital 
Table I. Characteristics of EGFR-mutated NSCLC patients with or without acid suppressant (AS) treatment.

\begin{tabular}{|c|c|c|c|}
\hline Characteristics & $\begin{array}{c}\text { With AS } \\
(n=11)\end{array}$ & $\begin{array}{l}\text { Without AS } \\
\quad(n=35)\end{array}$ & P-value \\
\hline Age, years [median (range)] & $74(52-88)$ & $77(52-89)$ & 0.708 \\
\hline Gender & & & 1.000 \\
\hline Male & 2 & 9 & \\
\hline Female & 9 & 26 & \\
\hline Smoking history & & & 0.559 \\
\hline Never smokers & 11 & 31 & \\
\hline Current/former smoker & 0 & 4 & \\
\hline ECOG performance status & & & 0.433 \\
\hline $0-1$ & 10 & 31 & \\
\hline $2-4$ & 1 & 4 & \\
\hline Histology & & & 0.138 \\
\hline Adenocarcinoma & 9 & 34 & \\
\hline Others & 2 & 1 & \\
\hline Stage & & & 1.000 \\
\hline IIIA & 2 & 8 & \\
\hline IV & 9 & 27 & \\
\hline Body surface area, $\mathrm{m}^{2}$ [median (range)] & $1.42(1.11-1.86)$ & $1.41(1.17-1.83)$ & 0.588 \\
\hline EGFR mutation & & & 0.133 \\
\hline Exon 19 deletion & 9 & 15 & \\
\hline Exon21 L858R & 2 & 18 & \\
\hline Others & 0 & 2 & \\
\hline
\end{tabular}

EGFR, epidermal growth factor receptor; NSCLC, non-small-cell lung cancer; ECOG, Eastern Cooperative Oncology Group.

and the Mito Medical Center between September, 2005 and May, 2013, were retrospectively analyzed. The histopathological diagnoses were performed according to the World Health Organization classification system (8) and the patients were staged according to the Union for International Cancer Control tumor-node-metastasis staging system (9).

The patient characteristics and the treatment efficacy and safety were evaluated using patient data extracted from the database of each participating institution. Tumor response was classified as complete response, partial response, stable disease, progressive disease or not evaluable, according to the Response Evaluation Criteria in Solid Tumors, version 1.1 (10).

We next evaluated the TKI dose prescribed and patient survival, taking body surface area (BSA) into consideration. Briefly, we defined relative dose of TKI as the prescribed dose divided by the full dose (gefitinib, $250 \mathrm{mg}$; erlotinib, $150 \mathrm{mg}$ ). In addition, we divided the relative dose of TKI by BSA and evaluated the association between relative dose of TKI/BSA and survival of patients with or without AS treatment.

The present retrospective study conformed to the Ethical Guidelines for Clinical Studies issued by the Ministry of Health, Labor and Welfare of Japan. Ethical approval of this study was obtained from the Institutional Review Board of the Ryugasaki Saiseikai Hospital (Ryugasaki, Japan).

Statistical analysis. Statistical significance was determined using the Mann-Whitney U test and the Chi-square test.
Survival time was calculated from the day of TKI initiation to death or last follow-up. The survival rate was analyzed with the Kaplan-Meier method and comparisons were performed using the log-rank test. $\mathrm{P}<0.05$ was considered to indicate a statistically significant difference.

\section{Results}

Patient characteristics. During the study period, a total of 46 patients were treated with TKIs (29 with gefitinib and 17 with erlotinib). Of the 46 patients, 11 received AS treatment and 35 did not. The characteristics of EGFR-mutated NSCLC patients with or without AS treatment are summarized in Table I. As regards age, gender, smoking history, performance status, cancer histology, clinical stage, BSA and type of EGFR mutation, there were no statistically differences between the two groups.

TKI therapy. The characteristics of TKI therapy are shown in Table II. There were no statistically significant differences in terms of TKI prescribed, dose reduction, prior therapy and relative dose of TKI/BSA between patients with and those without AS treatment.

Survival analysis. There were no statistically significant differences in PFS between the 11 patients with AS and the 35 patients without AS treatment (Fig. 1) $(\mathrm{P}=0.296)$. OS also did not differ significantly between the two groups (Fig. 2) $(\mathrm{P}=0.613)$. 
Table II. Prescribed TKIs, dose reduction, prior therapy and $\%$ of initial dose/BSA in patients with or without AS treatment.

\begin{tabular}{|c|c|c|c|}
\hline Variables & With AS & Without AS & P-value \\
\hline Prescribed TKI (patient no.) & & & 0.501 \\
\hline Gefitinib & 8 & 21 & \\
\hline Erlotinib & 3 & 14 & \\
\hline TKI dose reduction & & & 0.2419 \\
\hline Present & 2 & 13 & \\
\hline Absent & 9 & 22 & \\
\hline Prior therapy & & & 0.282 \\
\hline Present & 4 & 14 & \\
\hline Absent & 7 & 21 & \\
\hline$\%$ of initial dose/BSA $\left(\% / \mathrm{m}^{2}\right)$ & & & 0.471 \\
\hline Median (range) & $70.4(48.9-90.1)$ & $70.9(47.5-85.5)$ & \\
\hline
\end{tabular}

TKI, tyrosine kinase inhibitor; BSA, body surface area; AS, acid suppressant.

Table III. Relative dose of TKI/BSA and survival in patients with or without AS treatment.

\begin{tabular}{|c|c|c|c|}
\hline \multirow[b]{2}{*}{ Relative dose of TKI ${ }^{\mathrm{a}} / \mathrm{BSA}$} & \multicolumn{2}{|c|}{ Survival } & \multirow[b]{2}{*}{ P-value } \\
\hline & With AS & Without AS & \\
\hline \multicolumn{4}{|l|}{$\geq 70\left(\% / \mathrm{m}^{2}\right)$} \\
\hline Number of patients & 5 & 17 & \\
\hline PFS, months (range) & 24 (1-NA) & 18 (2-NA) & 0.959 \\
\hline OS, months (range) & 44 (1-NA) & 34 (17-NA) & 0.342 \\
\hline \multicolumn{4}{|l|}{$<70\left(\% / \mathrm{m}^{2}\right)$} \\
\hline Number of patients & 6 & 18 & \\
\hline PFS, months (range) & $6(1-N A)$ & $13(2-29)$ & 0.959 \\
\hline OS, months (range) & $4(1-N A)$ & 22 (11-NA) & 0.361 \\
\hline
\end{tabular}

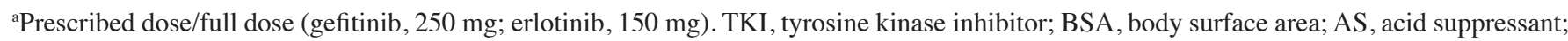
PFS, progression-free survival; OS, overall survival; NA, not applicable.

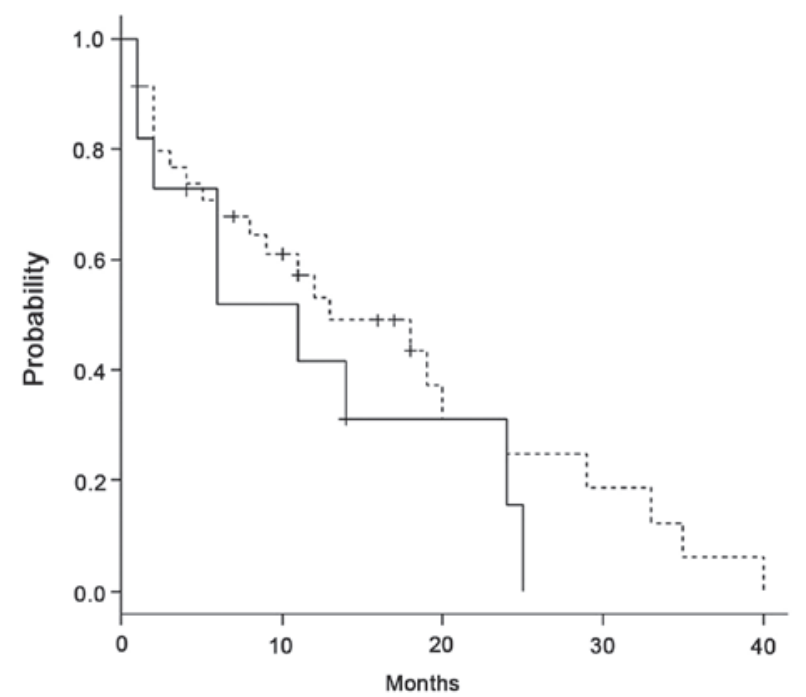

Figure 1. Progression-free survival curves of epidermal growth factor receptor-mutated non-small-cell cancer patients with or without acid supressant (AS) treatment. Solid line, patients with AS; dashed line, patients without AS.

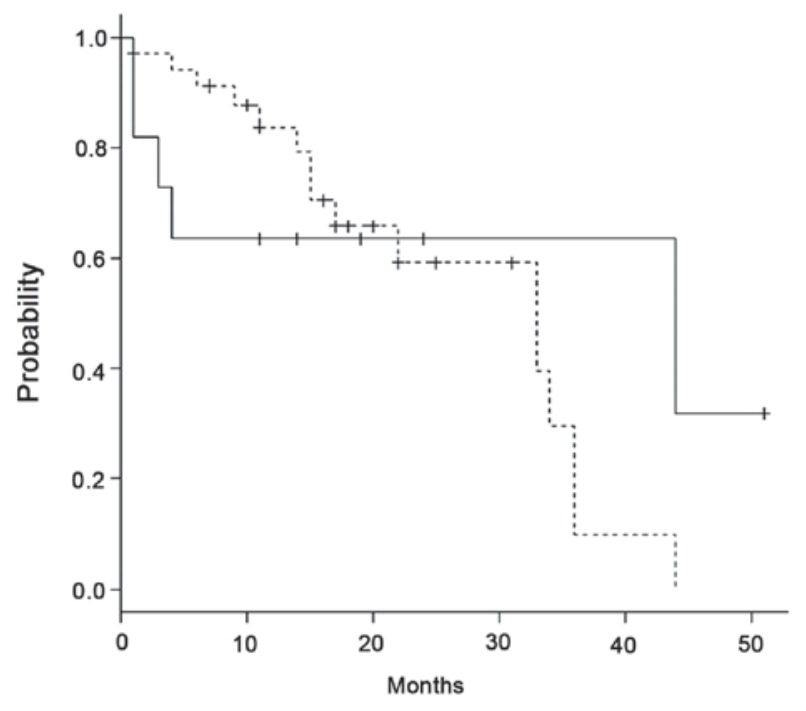

Figure 2. Overall survival curves of epidermal growth factor receptor-mutated non-small-cell cancer patients with or without acid supressant (AS) treatment. Solid line, patients with AS; dashed line, patients without AS. 
Association between relative dose of TKI/BSA and survival. We next evaluated the association between the relative dose of TKI/BSA and survival in NSCLC patients with or without AS treatment. The median of the relative dose of TKI/BSA in patients with AS and those without AS was 70.4 and $70.9 \% / \mathrm{m}^{2}$, respectively (Table II); therefore, we performed the analysis in patients with a relative dose of TKI/BSA of $\geq 70$ and in those with a relative dose of TKI/BSA of $<70 \% / \mathrm{m}^{2}$. There were no statistically significant differences in PFS and OS between the two groups of patients (Table III).

\section{Discussion}

In the present study, we evaluated whether oral AS therapy is truly associated with decreased efficacy of gefitinib and erlotinib, particularly in patients with mutated EGFR. Our results demonstrated that oral AS therapy is not associated with decreased efficacy of gefitinib and erlotinib in patients with mutated EGFR, which was inconsistent (5) or consistent (6) with previous studies. Chu et al (5) reported that gastric acid suppression was associated with decreased erlotinib efficacy in NSCLC patients. That retrospective study included 507 patients with advanced NSCLC, but there were 487 patients with unknown EGFR mutation status. By contrast, Hilton et al (6) reported that the co-administration of AS treatment with erlotinib did not appear to exert a significant effect on the median plasma drug levels or the outcome. The study evaluated pharmacokinetics and survival in the patients of a phase III clinical trial (BR.21), but there was no information regarding the association among AS treatment, EGFR mutation status and patient survival (6). Although it is clear that AS administration may affect the absorption of erlotinib in an experimental setting, it is uncertain whether the decreased erlotinib efficacy associated with AS in the study by Chu et al (5) can be generalized when AS agents are prescribed for oral use, or when other TKIs, such as gefitinib, are prescribed in patients with mutated EGFR. This inconsistence in the results may be attributed to a number of possible reasons, such as the difference in EGFR mutation status, difference in BSA of the patients, difference in the AS agent prescribed, and different ethnicities. All our patients had mutated EGFR, the median BSA of the patients was 1.41-1.42 $\mathrm{m}^{2}$, all the patients had received oral AS treatment, and all our patients were Asians and Caucasians similar to the study by Chu et al (5).

It is well known that the association between the maximum tolerated dose (MTD) and recommended dose (RD) in erlotinib and gefitinib treatment may be associated with this inconsistency. The RD of gefitinib (250 mg/day) for NSCLC is only one-third of its MTD, whereas the RD used for erlotinib $(150 \mathrm{mg} / \mathrm{day})$ is its MTD $(11,12)$. Cigarette smoking is a well-known factor that may alter erlotinib plasma levels (13). In our study, there was no difference in smoking status between patients with and those without AS treatment. In addition, no patients were smoking during the TKI administration. Another explanation for the inconsistency may be that it reflects the presence of comorbidities where AS use was indicated, such as aspirin for cardiovascular disease, dexamethasone for symptomatic NSCLC, gastroesophageal reflux or treatment for duodenal or gastric ulcers. Taking this evidence into consideration, we hypothesized that, in patients with mutated EGFR, decreased AUC by AS therapy may exert a relatively smaller effect on TKI efficacy compared with that in patients with wild-type EGFR, i.e., prevalence of an EGFR mutation may allow a significantly lower than standard TKI dose to be effective, thereby circumventing the reduced TKI absorption caused by AS therapy, as suggested by Yeo et al in a study on erlotinib therapy (14).

The main limitation of our study is due to its retrospective design and the small sample size. In addition, there were no data on pharmacokinetics and its effect on treatment efficacy. In addition, no data are available on whether AS treatment decreases TKI serum levels in Japanese patients. If so, it is not known whether the serum level is decreased below what would be clinically efficient. However, despite these limitations, the difference in our outcomes compared with those of previous studies on erlotinib therapy may be of clinical value when administering TKIs and AS therapy in NSCLC patients with mutated EGFR.

In conclusion, our study suggests that AS treatment may not compromise TKI efficacy in patients with mutated EGFR. Prospective studies and large-scale confirmation studies investigating the effect of AS and TKI co-administration in NSCLC patients with mutated EGFR may be meaningful in clinical practice.

\section{References}

1. Achem SR and DeVault KR: Gastroesophageal reflux disease and the elderly. Gastroenterol Clin North Am 43: 147-160, 2014.

2. Keynes WM: Medical response to mental stress. J R Soc Med 87: 536-539, 1994.

3. US Food and Drug Administration: Center for Drug Evaluation and Research. Tarceva NDA 21-743/S000 clinical pharmacology and biopharmaceutics review, November 18, 2004. Available at: http://www.accessdata.fda.gov/drugsatfda_docs/ nda/2004/21-743_Tarceva_biopharmr.PDF. Accessed: May 1, 2014.

4. Budha NR, Frymoyer A, Smelick GS, Jin JY, Yago MR, Dresser MJ, Holden SN, Benet LZ and Ware JA: Drug absorption interactions between oral targeted anticancer agents and PPIs: Is $\mathrm{pH}$-dependent solubility the Achilles heel of targeted therapy? Clin Pharmacol Ther 92: 203-213, 2012.

5. Chu MP, Ghosh S, Chambers CR, Basappa N, Butts CA, Chu Q, Fenton D, Joy AA, Sangha R, Smylie M and Sawyer MB: Gastric acid suppression is associated with decreased erlotinib efficacy in non-small-cell lung cancer. Clin Lung Cancer 16: 33-39, 2015.

6. Hilton JF, Tu D, Seymour L, Shepherd FA and Bradbury PA: An evaluation of the possible interaction of gastric acid suppressing medication and the EGFR tyrosine kinase inhibitor erlotinib. Lung Cancer 82: 136-142, 2013.

7. Ter Heine R, Fang giday JC, Lankheet NA, Beijnen JH, Van Der Westerlaken MM, Staaks GH and Malingré MM: Erlotinib and pantoprazole: A relevant interaction or not? Br J Clin Pharmacol 70: 908-911, 2010.

8. Travis WD, Brambilla E, Muller-Hermelink HK and Harris CC (eds): Pathology and genetics: Tumours of the Lung, Pleura, Thymus and Heart. IARC Press, Lyon, pp9-122, 2004.

9. Vallières E, Shepherd FA, Crowley J, et al; International Association for the Study of Lung Cancer International Staging Committee and Participating Institutions: The IASLC Lung Cancer Staging Project: Proposals regarding the relevance of TNM in the pathologic staging of small cell lung cancer in the forthcoming (seventh) edition of the TNM classification for lung cancer. J Thorac Oncol 4: 1049-1059, 2009.

10. Eisenhauer EA, Therasse P, Bogaerts J, Schwartz LH, Sargent D, Ford R, Dancey J, Arbuck S, Gwyther S, Mooney M, et al: New response evaluation criteria in solid tumours: Revised RECIST guideline (version 1.1). Eur J Cancer 45: 228-247, 2009. 
11. Costa DB, Nguyen KS, Cho BC, Sequist LV, Jackman DM, Riely GJ, Yeap BY, Halmos B, Kim JH, Jänne PA, et al: Effects of erlotinib in EGFR mutated non-small cell lung cancers with resistance to gefitinib. Clin Cancer Res 14: 7060-7077, 2008.

12. Rukazenkov Y, Speake G, Marshall G, Anderton J, Davies BR, Wilkinson RW, Mark Hickinson D and Swaisland A: Epidermal growth factor receptor tyrosine kinase inhibitors: Similar but different? Anticancer Drugs 20: 856-866, 2009.
13. O'Malley M, King AN, Conte M, Ellingrod VL and Ramnath N: Effects of cigarette smoking on metabolism and effectiveness of systemic therapy for lung cancer. J Thorac Oncol 9: 917-926, 2014.

14. Yeo WL, Riely GJ, Yeap BY, Lau MW, Warner JL, Bodio K, Huberman MS, Kris MG, Tenen DG, Pao W, et al: Erlotinib at a dose of $25 \mathrm{mg}$ daily for non-small-cell lung cancers with EGFR mutations. J Thorac Oncol 5: 1048-1053, 2010. 